\title{
Interfacial Segregation in Modified Fe-Nd-B Permanent Magnets by Analytical Electron Microscopy
}

\author{
J. Bentley* and J.E. Shield** \\ *Metals \& Ceramics Div., Oak Ridge National Laboratory, PO Box 2008, Oak Ridge, TN 37831-6064 \\ **Dept. of Mechanical Engineering, University of Nebraska, Lincoln, NE 68588-0656
}

In fine-grained (<100 nm dia.) permanent magnet materials, intergranular exchange coupling can lead to "exchange-spring" behavior with enhanced remanance and energy product [1]. However, intergranular segregation can lead to undesirable properties due to magnetic isolation of individual grains. In this study, magnetic properties of modified Fe-Nd-B alloys were correlated to segregation measured by high-spatial-resolution analytical electron microscopy (AEM). Three alloy compositions were investigated: a ternary alloy with nominal composition (wt\%) $72.3 \mathrm{Fe}, 26.7 \mathrm{Nd}$, $1 \mathrm{~B}\left(\mathrm{Fe}_{14} \mathrm{Nd}_{2} \mathrm{~B}\right.$ stoichiometry); an alloy with $2.5 \mathrm{wt} \% \mathrm{Ti}$ and $0.6 \mathrm{wt} \% \mathrm{C}$ (TiC) added to the stoichiometric alloy; and a nine component alloy of composition (wt\%) 60.2 Fe, 6.9 Co, $14.8 \mathrm{Nd}, 9.9$ Pr, 3.0 Dy, 1.2 B, 2.4 Ti, 0.8 Zr, and $0.8 \mathrm{C}$ (which is slightly transition-metal-poor relative to the 14:2:1 stoichiometry). To produce fine-grained materials, arc-melted buttons were melt spun at 30 $\mathrm{m} / \mathrm{s}$ in 1 atmosphere of ultra-high-purity Ar to produce metallic glass ribbons (the ternary alloy was $\sim 5 \%$ crystalline) which were subsequently crystallized by annealing under conditions selected on the basis of differential thermal analysis (DTA). Ta-foil-wrapped samples were annealed in Ar-filled, sealed, quartz ampules at 565,626 and $631^{\circ} \mathrm{C}\left(5^{\circ} \mathrm{C}\right.$ above the respective crystallization onset temperatures) for 16, 26, and 6 min (time to $100 \%$ crystallinity as evidenced by the absence of an exothermic peak) for the ternary, quinternary and nine-component alloys, respectively. Specimens for AEM were prepared from ribbon pieces mounted on $\mathrm{Cu}$ slotted washers by ion milling at $4.5 \mathrm{kV}$ using a liquid-nitrogen-cooled stage, typically for 7-8 h. Initial characterization was performed with a JEOL 2000FXII and composition profiles were obtained with a Philips CM200-FEG equipped with an Emispec Vision system, an Oxford x-ray energy-dispersive spectrometer (EDS), and a Gatan imaging filter (GIF) operated in spectroscopy mode to record electron energy-loss spectra (EELS). A current of $1.2 \mathrm{nA}$ in a $1.5 \mathrm{~nm}$ (FWHM) probe, and dwell times per pixel of 10s were typically used to measure composition profiles.

The microstructures of the crystallized alloys are shown in figure 1. Measurements by AEM revealed no detectable changes in composition across grain boundaries in the ternary alloy. However, intergranular segregation was detected in the quinternary and nine-component alloys. Figure 2 shows typical results. Strong Ti enrichment was measured by EDS, along with slight enrichment of the rare earth elements and a weaker depletion of $\mathrm{Fe}$ and Co. There was some indication of intergranular $\mathrm{C}$ segregation. Enrichment of $\mathrm{Ti}$ (and $\mathrm{Zr}$ ) in front of the growing crystal interface in partially crystallized samples was also evident in spectrum lines and images. For quantification, self-consistent k-factors were obtained from the specimens themselves, assuming the nominal alloy compositions. Data from EELS recorded simultaneously with the EDS data yielded similar compositions.

This work has shown that the addition of alloying elements improved the glass-forming ability of $\mathrm{Fe}-\mathrm{Nd}-\mathrm{B}$ alloys and also improved the microstructural development during devitrification resulting in finer and more uniformly sized grains. The grain refinement was most likely the result of elemental rejection from the growing grains during crystallization and the consequent development of intergranular segregation. Correlation with measured magnetic properties revealed that the more uniform microstructures of the modified alloys resulted in squarer demagnetization behavior and a smaller distribution of switching fields. However, the segregation in the modified alloys partly isolated the grains, inhibiting intergranular exchange coupling and increasing the coercivity. Thus, alloying induced more uniform and refined nanostructures critical to exchange-spring permanent magnets, but inhibited intergranular exchange interactions, thereby negating the effects of finer grain 
size. Through optimal heat treatments it may be possible to reduce the segregation or cause precipitation (e.g. TiC), resulting in "cleaner" grain boundaries and enhanced intergranular exchange interactions [2].

References

[1] E.F. Kneller and R. Hawig, IEEE Trans. Magn. 27 (1991) 3588.

[2] The contributions of B.B. Kappes (University of Utah, Salt Lake City, UT 84112) and D.J. Branagen (Idaho National Engineering and Environmental Laboratory, Idaho Falls, ID 83415) are gratefully acknowledged. This research was supported in part by the National Science Foundation through grant no. DMR9714946 and at the ORNL SHaRE Collaborative Research Center by the Division of Materials Sciences and Engineering, U.S. Department of Energy, under contract DE-AC05-00OR22725 with UT-Battelle, LLC.
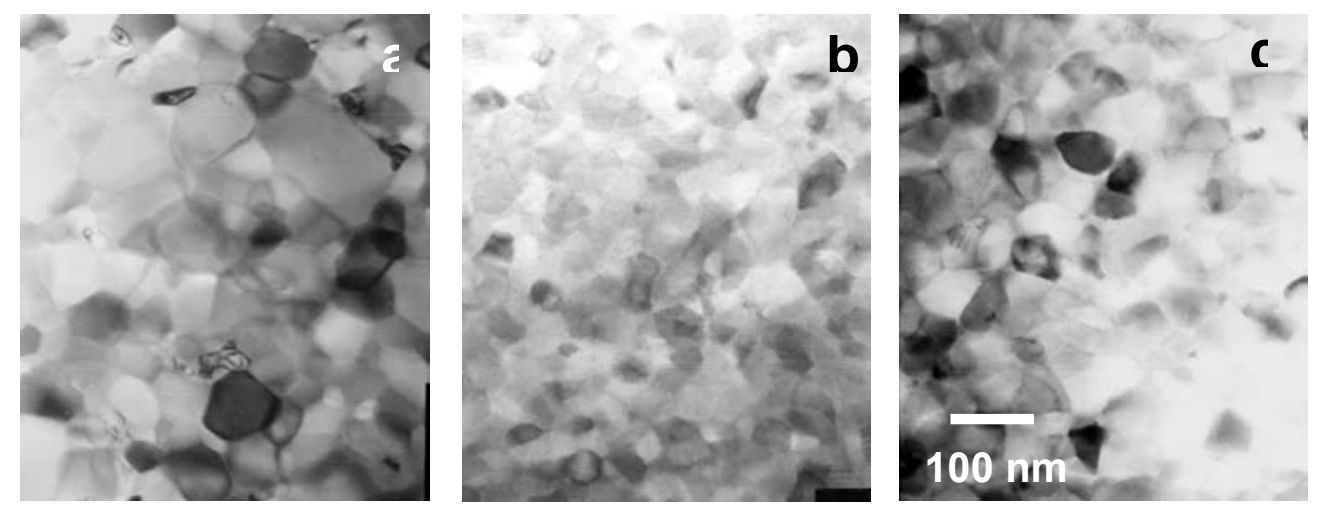

FIG. 1. TEM bright field micrographs of crystallized (a) Nd-Fe-B, (b) Nd-Fe-B-Ti-C, and (c) Nd-PrDy-Fe-Co-B-Ti-Zr-C alloys. Grain sizes were (a) $77 \pm 30 \mathrm{~nm}$, (b) $45 \pm 12 \mathrm{~nm}$, and (c) $60 \pm 13 \mathrm{~nm}$.

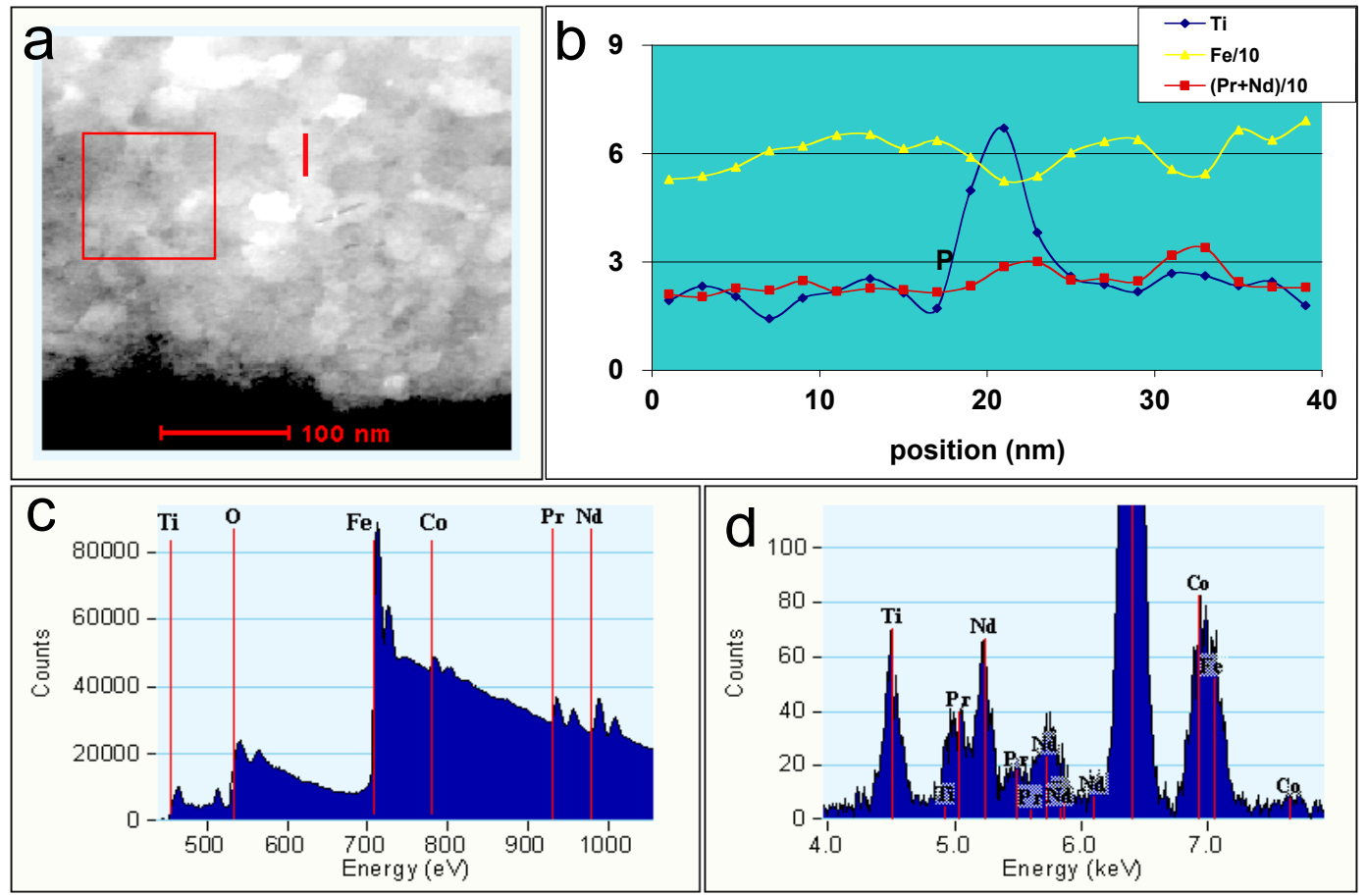

FIG. 2 Composition profile in Nd-Pr-Dy-Fe-Co-B-Ti-Zr-C alloy. (a) DF-STEM image with red line indicating position of 40-nm profile across grain boundary. (b) Composition profiles from EDS data. (c) Background-subtracted EELS data (intragranular). (d) Partial EDS data (intergranular). 